\title{
Primitive-based Teleconference Image Coding Technique
}

\author{
Adnan M. Alattar \\ and \\ Sarah Rajala
}

Center for Communications and Signal Processing Electrical and Computer Engineering Department North Carolina State University

CCSP-TR-88/25

October, 1988 


\title{
Primitive-Based Teleconference Image Coding Technique
}

\author{
Adnan M. Alattar and Sarah A. Rajala \\ Center for Communications and Signal Processing \\ P.O. Box 7911, North Carolina State University \\ Raleigh, NC 27695-7914
}

\begin{abstract}
In this paper a new image coding technique that has the potential of producing good image quality at a very high compression ratio is described. The new technique is best suited for coding teleconference and Picture Phone images, which require a compression ratio on the order of 1000:1 when transmitted through a low bit rate channel of $64 \mathrm{kbits} / \mathrm{s}$. The main idea behind this new technique is to break the image into complicated primitives using a priori knowledge about the image. These primitives are not necessarily coded and transmitted. Instead, they are matched to a previously created database, and the necessary information about the best match is coded and transmitted. This information is used at the receiver to retrieve a replica of the original primitive from the same database. Eventually, all primitives are put together as in computer animation to produce a faithful reconstruction of the original image. Basically, the coder consists of a primitive extractor followed by a primitive matcher that interacts with a database to produce a sequence of messages. The messages are coded and transmitted through the channel. The primitive extractor also interacts with a factbase which contains a priori information about the image formation. The database is a collection of the primitives in the images for a certain application. The quality of images produced from this technique is very good at an extremely high compression ratio.
\end{abstract}

\section{Introduction}

The idea of composing pictures from small pieces of other pictures has long been used in applications such as animation [1], forensic [2] and graphics [3]. In animation, characters or pieces of a character are hand-drawn on a clear plastic called cellulose, or cel for short. The cels are stacked on top of each other, then photographed to create one frame. To produce motion, an animator creates successive frames by changing or moving the cels of the stack and photographing the new stack. For example, the animator changes the facial expressions to give the illusion that a character in the picture is speaking. Today, computers are used to assist in producing animated 
films [4].

In forensic applications, a special kit of facial features is used to construct a picture of a criminal from the memory of a witness. Photofit, Identikit, Magnaface, Videofit, and Minolta Montage Synthesizer are some of the commercial kits that are used by the police in criminal identification [2]. Photofit and Identikit utilize approximately 560 variations of facial features, such as eyes, noses, mouths, chins, and hair sections. These features have been abstracted from monochrome photographs. Magnaface and Videofit are recent developments that produce realistic portraits in full color. The Minolta Montage Synthesizer is a device produced in Japan for constructing facial images by blending features from different photographs. It has three parts: an optical blender, a closed circuit television camera and a television monitor. The blender enables partial images from separate sources to be combined, thus producing a composite image.

In computer graphics, a system called Whatsisface has been developed by Gillenson and Chandrasekaran [3] to draw human faces on a graphic display. The system has pre-stored line-drawings of facial features, an average face as a starting point, and a heuristic strategy that guides the user in the construction of the facial image. The heuristic strategy begins by displaying an average face which the user stretches horizontally and vertically to approximate the shape of the new image. The user also updates the features in the image by retrieving suitable features from a database. 
In this paper, the idea of constructing images using pre-stored image segments is extended to image coding. The new primitive-based coder produces very good images with a very high compression ratio, making it suitable for teleconferencing and picture phone applications. The paper is divided into five sections. Section (2) discusses the general coding methodology. Section (3) discusses the block diagram of both the coder and the decoder of the new technique. Section (4) discusses the application of the new technique for teleconference and picture phone images. The last section is a conclusion.

\section{Primitive-Based Image Coding Technique}

Most of the traditional image coding techniques follow one of two basic coding strategies: transform or predictive [5]. Both techniques concentrate on coding messages that are algebraically calculated from the pixel values of the image. Hence, these techniques may be classified as algebraic techniques. More recently, a new class of techniques, known as second generation techniques [6,7], have utilized image segments of uniform properties as messages rather than numerical messages [6,7]. The achievable compression ratio from these second generation techniques is amazingly high (the order of 100:1) compared to those obtained from traditional techniques (maximum 20:1).

The new technique presented in this paper is an extension of the second generation techniques. It achieves a very high compression ratio (over 500:1) by using high level primitives instead of segments of uniform properties. As was previously 
mentioned, any image can be broken into a set of disjoint primitives, each of which represents a small object or feature of the original image. For example, an image of a human face can be broken into regions defining physical features, such as the eye, mouth, and nose. These features may be considered to be primitives of such an image, where each primitive may have more than one state. For instance, the mouth may be closed, slightly open, open or widely open.

For each class of images, there is a universal set of primitives $\mathbf{A}$, of which the set of primitives $\mathbf{B}_{\mathbf{f}}$ in any image $f$ of that class is a subset. If $\mathbf{B}_{f}=\left\{b_{1}{ }^{\left(j_{1}\right)}, b_{2}{ }^{\left(j_{2}\right)}, \ldots, b_{n-1}{ }^{\left(j_{n-1}\right)}, b_{n}{ }^{\left(j_{n}\right)}\right\}$, where $j_{i}$ indicates the state of the $i^{\text {th }}$ primitive $b_{i}$, then the image $f$ can be represented as the union of its primitives; i.e,

$$
f=\bigcup_{i=1}^{n} b_{i}^{\left(j_{i}\right)}
$$

Equation (1) indicates that, if the set $\mathbf{A}$ is known and the subset $\mathbf{B}_{f}$ of each image $f$ is identified from $\mathbf{A}$, then the image $f$ can be easily constructed using the set $\mathbf{A}$. If we assume that the type of primitive that appears in a given application is finite, $\mathbf{A}$ is a finite set that can be constructed from prototype images. When $A$ is known, the primitives of any image in the specified application can be specified in terms of those in $\mathbf{A}$ and some information about the size, orientation, and location of the original primitives.

In the new primitive-based coding technique, a database that represents the set A is assumed to be available before coding the image. The entries in the database are extracted from prototype images that best represent the specified application. The 
primitives in the database are normalized with respect to size and orientation. This normalization is essential to the subsequent matching process between the original primitives and the entries in the database. The coding process starts by extracting the primitives $\mathrm{B}_{f}$ of the image, using image analysis techniques. Then, these primitives are normalized with respect to size and orientation, and are matched to the database A. When the best matches are found, their order in the database, normalization factors and locations in the original image are coded and transmitted. If no good match for a primitive is found, the exact primitive is transmitted and the databases in both transmitter and receiver are updated to include the new primitive.

The receiver uses the transmitted information and the same database to reconstruct a faithful replica of the original image. The decoding process is similar to the idea used in computer animation. First, each primitive is extracted from the database using its order; it is then scaled and oriented using its normalization factors so that it assumes its original size and orientation. Finally, each primitive is projected onto its original place in the image.

For time-varying image sequences, it is not necessary to send information about every primitive in the image; instead, it is sufficient to send information about only those primitives that encounter significant changes, such as rotation, scaling or deformation, from one frame to the next. In this case, projection of the primitives is achieved by assigning each primitive a full opaqueness level then adding it to the base image at the original location of the primitive. The base image is the previous frame 
which is assumed to be known to the receiver. Image compression is achieved in this technique in two ways. The first is by coding only those primitives that encounter significant changes. The second is by sending a small number of parameters (the order of a primitive in the database, its original location in the image and its normalization factors) instead of sending the primitive itself.

\section{The Structure of The Coder and Decoder}

A block diagram for the new coding technique is shown in Fig. 1. It consists of eight basic blocks: the primitive extractor, factbase, database, database updator, primitive matcher, primitive coder, the binary coder and the controller which maintain the entire system. The primitive extractor breaks the image into image primitives, each of which forms a message. The pixels inside a primitive are not necessarily uniform with respect to any property, but need to represent a meaningful feature or symbol. Hence, the message in this case is more complicated than a simple segment of uniform characteristics. The primitive extractor may use a priori knowledge to locate and extract the primitives in the image. A priori knowledge such as type of image to be coded, image formation model, primitive location model, and primitive motion model is stored in the factbase. This factbase is accessible to the primitive extractor.

The primitive matcher finds the best match to a primitive from the database. The database is a collection of many image segments that might appear in an image in a given application. Its contents are application dependent. For instance, for head 
and shoulder images, the database contains pictures of various eyes, noses, mouths, hair segments and ears; for FAX purposes, it could contain the alpha-numerical characters of many fonts and basic graphics primitives. In all cases, however, the database is assumed to be available before coding any picture.

The coder's controller coordinates and synchronizes the operations of other blocks. It first determines the operation mode based on the input image, and tells the primitive extractor which facts from the factbase are to be used in extracting the primitives of the input image. It also manages the database through the database update routine and decides how a primitive is coded by the primitive coder. The binary coder codes and transmits the required information produced by the primitive coder.

The decoder operates in full synchronization with the coder, and its structure is similar to that of the coder. A block diagram of the decoder is shown in Fig. 2. The database at the decoder is exactly the same as that used by the coder. The primitive decoder extracts the suitable primitives from the database. The primitive projector reverses the action of the message extractor of the coder. It takes a primitive and its normalization and location factors as inputs, scales and directs the primitive to its original size and orientation, and then projects it onto its original location in the picture. Finally smoothing is used to remove any artifacts that might appear in the transition regions between the primitives after decoding the image. 


\section{Video Teleconferencing and Picture Phone Images}

Video teleconferencing and Picture Phone are two major applications of the new coding technique. Head and shoulder images are typical in both applications, and the motion in those images is limited to the movement of the head, eyes, mouth and jaws, and hands (if hands appear in the picture). The motion of these features tends to be repetitive; hence, the primitives assume a small number of states that are easily collected in a database. In the following subsection, the a priori knowledge about head and shoulder images that is useful for feature extraction is used in constructing an image model.

\subsection{Modeling The Head and Shoulder Image}

The head of the human being can be thought of as an ellipsoid that sits on the top of the torso, and is attached to it from the center by the neck. Hence, the head movement can be viewed as rotations $\alpha, \beta$, and $\theta$ about $x, y$, and $z$ axes, respectively, which pass through the center of the ellipsoid (see Fig. 3). Viewed from most angles, the head looks like an ellipse. The elongation and orientation of this ellipse are related to the head rotation angles $\alpha, \beta$ and $\theta$. For example, the length of the minor axis is about two thirds that of the major axis in the normal front view position $[8,9]$. This ratio changes as the head moves up or down, and hence, it is related to the value of the angle $\alpha$ (assuming the $x-y$ plane is the image plane). The angle $\theta$ is directly indicated by the direction of the minor axis of the ellipse. The angle $\beta$ is related to 
the symmetry of the head view in the original image.

Only the eyes, nose, and mouth are significant to the new coding methodology. The local orientation of these primitives is related to the global orientation of the head. In fact, the shapes of these features are highly dependent on the rotation angle $\beta$. If the head is rotated to the left side, then the left eye and ear do not appear in the image, and the mouth, nose and right eye have side views. In the frontal view of the head (the normal case) the angle $\beta$ is zero, and the effect of angle $\alpha$ is not that significant; hence it can be ignored.

\subsection{Extracting The Facial Features}

The location of the three most important features: eyes, nose and mouth can easily be determined. It is well known in the art of drawing the human head that the head is approximatelly five eye-lengths wide [8,9] (see Fig. 4). Both eyes lie on the midway line, and the distance between them is equal to almost one eye-length. The nose starts at the center of the face and descends to a point mid-way between the bridge of the nose and the base of the chin. The width of the nose at its base is also equal to one eye-length. The mouth barrel starts at the nose and extends two thirds of the distance down from the nose to the chin. The sides of the barrel align with the centers of the eye sockets. These measurements are used to locate the head features when the location and orientation of the head are known. 
The global orientation of the head is determined from the head view in the image. This view has an elliptic shape under the ellipsoid model of the head. The parameters of the ellipse are estimated from the points on the edge of the head by fitting an ellipse through these points. A simple way to do this is by first extracting the contour of the head and then minimizing the fitting error. The quadratic form for an ellipse is given by,

$$
f(x, y ; a, b, c, d, e)=a x^{2}+2 b x y+c y^{2}+d x+e y-1=0
$$

and the fitting error is defined by

$$
S=\sum_{i=1}^{n}\left(\Delta x_{i}\right)^{2}+\left(\Delta y_{i}\right)^{2}
$$

where $\Delta x_{i}$ and $\Delta y_{i}$ are the $x$ and $y$ deviations of the point $\left(x_{i}, y_{i}\right)$ from the ellipse contour.

Let $\left(c_{x}, c_{y}\right)$ be the center of the ellipse that fits the head contour, and let $l_{x}, l_{y}$ and $\theta$ denote the minor and major semi-axes and orientation of the ellipse, respectively. Using the previously described model of the head, the location of the eyes, nose, and mouth are given below in terms of $\left(c_{x}, c_{y}\right), \theta, l_{x}$, and $l_{y}$.

\section{Eyes:}

If the length of each eye is $e_{l}$ and the centers of right and left eyes are (cre $\left.e_{x}, c r e_{y}\right)$ and $\left(c l e_{x}, c l e_{y}\right)$, respectively, then,

$$
e_{l}=\frac{2 l_{x}}{5}
$$




$$
\begin{aligned}
& \operatorname{cre}_{x}=c x+e_{l} \cos \theta \\
& \operatorname{cre}_{y}=c y+e_{l} \sin \theta \\
& c l e_{x}=c x-e_{l} \cos \theta \\
& c l e_{y}=c y-e_{l} \sin \theta
\end{aligned}
$$

Nose:

If the center, length, and base width of the nose are denoted by $\left(n c_{x}, n c_{y}\right), n_{h}$ and $n_{w}$, respectively, then,

$$
\begin{gathered}
n c_{x}=c_{x}-\frac{l_{y}}{4} \sin \theta \\
n c_{y}=c_{y}+\frac{l_{y}}{4} \cos \theta \\
n_{h}=\frac{l_{y}}{2} \\
n_{w}=\frac{2 l_{x}}{5}
\end{gathered}
$$

Mouth:

If the center, height, and width of the mouth barrel are denoted by $\left(m c_{x}, m c_{y}\right)$, $m_{h}$ and $m_{w}$, respectively, then,

$$
\begin{aligned}
& m c_{x}=c_{x}-\frac{2 l_{y}}{3} \sin \theta \\
& m c_{y}=c_{y}+\frac{2 l_{y}}{3} \cos \theta
\end{aligned}
$$




$$
\begin{aligned}
& m_{h}=\frac{l_{y}}{3} \\
& m_{w}=\frac{4 l_{x}}{5}
\end{aligned}
$$

\subsection{Database and Primitives Matching}

The best match from the database is found by comparing the extracted feature with features of the same type from the database. The database for this class of images is very simple. The entries of the database are grouped under the feature types; hence there are groups labeled eyes, nose, and mouth. Each group (e.g. the eyes) contains various states that may be assumed by its corresponding feature (e.g. eye is closed, slightly opened, or fully opened). Since each feature in the original image is extracted by its name, a feature is matched to only one group. Mean-squareerror is used as a measure of matching error. Since there may be slight missalignment between the extracted feature and those in the database, it is necessary to displace the feature one or two pixels in each direction and compare it with those in the database. The feature with the least mean-square-error is taken as the best match.

The parameters of the ellipse, normalization factors of the primitives, and the order of the feature's best match are sent to the receiver, where the same database is used to reconstruct a faithful image replica. The decoding process starts by rotating the head in the previous frame by the appropriate angle, then updating the facial features from the database. 


\subsection{Implementation and Simulation Results}

The described Primitive-Based image coding technique was successfully implemented. The coder was simulated using head and shoulder images of the type shown in Fig. 5 and 6. The size of these images is $128 \times 128$ pixels, and each pixel is represented by 8 bits. The previous frame shown in Fig. 5 was assumed to be known to the receiver before coding the image of Fig. 6 .

To locate the eyes, nose and mouth, a Sobel edge detector [10] was used to find the edges in the image. These edges were thinned using the Chen et. al. thinning algorithm [11], and the ellipse which fit the head contours was estimated from the thinned contours. Finally, the three features (eyes, nose, and mouth) were extracted using the ellipse parameters and equations (4)-(6). The extracted features were matched to a simple database that was created using the same image sequence. The head of the previous frame was rotated, and the three primitives were projected. The transition regions around the head and primitives were smoothed using an average filter. The decoded image is shown in Fig. 7.

Only 80 bits were required to code the image of Fig. 6: 16 bits were allocated for the orientation of the ellipse and 8 hits for each of the other parameters. Hence, an incredibly high compression ratio of 1638:1 was achieved for a single frame. The image quality is very good in spite of the achieved compression ratio. The use of prestored image segments made the extremely high compression ratio feasible. Hence, there is a trade off between the compression ratio and the required memory storage. 
However, with the availability of cheap memory devices, memory requirement is not a limitation of the new technique.

\section{Conclusion}

The primitive-based image coding technique has the potential of producing very good image quality at an incredibly high compression ratio (over 1000:1). This high ratio is achieved by using a database of image primitives. The decoded image quality depends on the quality of the database in representing the images in a certain application. The new technique enables real-time transmission of video signals over low bit rate channels (less than $64 \mathrm{Kbits} / \mathrm{s}$ ). It can be used in coding head and shoulder images, such as teleconference, Picture Phone, and mug-shot images. The technique can also be extended to other applications, given a primitive extractor and a good database for those applications. 


\section{References}

[1] N. Magnenat-Thalmann and D. Thalmann, Computer Animation, SpringerVerlag, Tokyo 1985.

[2] G. Davies, H. Ellis, and J. Shepherd, Perceiving and Remembering Faces, Academic Press, London 1981.

[3] M.L. Gillenson and B. Chandrasekaran, "A Heuristic Strategy for Developing Human Facial Images on a CRT," Pattern Recognition, vol. 7, pp. 187-196, 1975.

[4] N. Burtnyk and M. Wein, "Computer-Generated Key-Frame Animation," J. of Society of Motion Picture and Television Engineers, vol. 80, pp. 149-153, 1971.

[5] A.N. Netravali and J.O. Limb, "Picture Coding: A review," Proc. IEEE, vol. 68, pp. 366-406, March 1980.

[6] M. Kunt, A. Konomopoulos and M. Kocher, "Second-Generation Image Coding," Proc. IEEE, vol. 73, pp. 549-574, April 1985.

[7] S.A. Rajala, M.R. Civanlar and W.M. Lee, "A Second Generation Image Coding Technique Using Human Visual System Based Segmentation," Proc. ICASSP'87, pp. 1362-1365, April 1987.,

[8] B. Hogarth, Drawing the Human Head, London 1975.

[9] S. Simmons III and M.S. Winer, The Creative Process, Prentice-Hall, New Jersey 1977.

[10] E.L. Hall, Computer Image Processing and Recognition, Academic Press, New York 1979.

[11] R.T. Chen, H.K. Wan, D.L. Stover, and R.D Iverson, "A One-Pass Thinning Algorithm and Its Parallel Implementation," Computer Vision, Graphics, and Image Processing, vol. 40, pp. 30-40, Oct. 1987. 


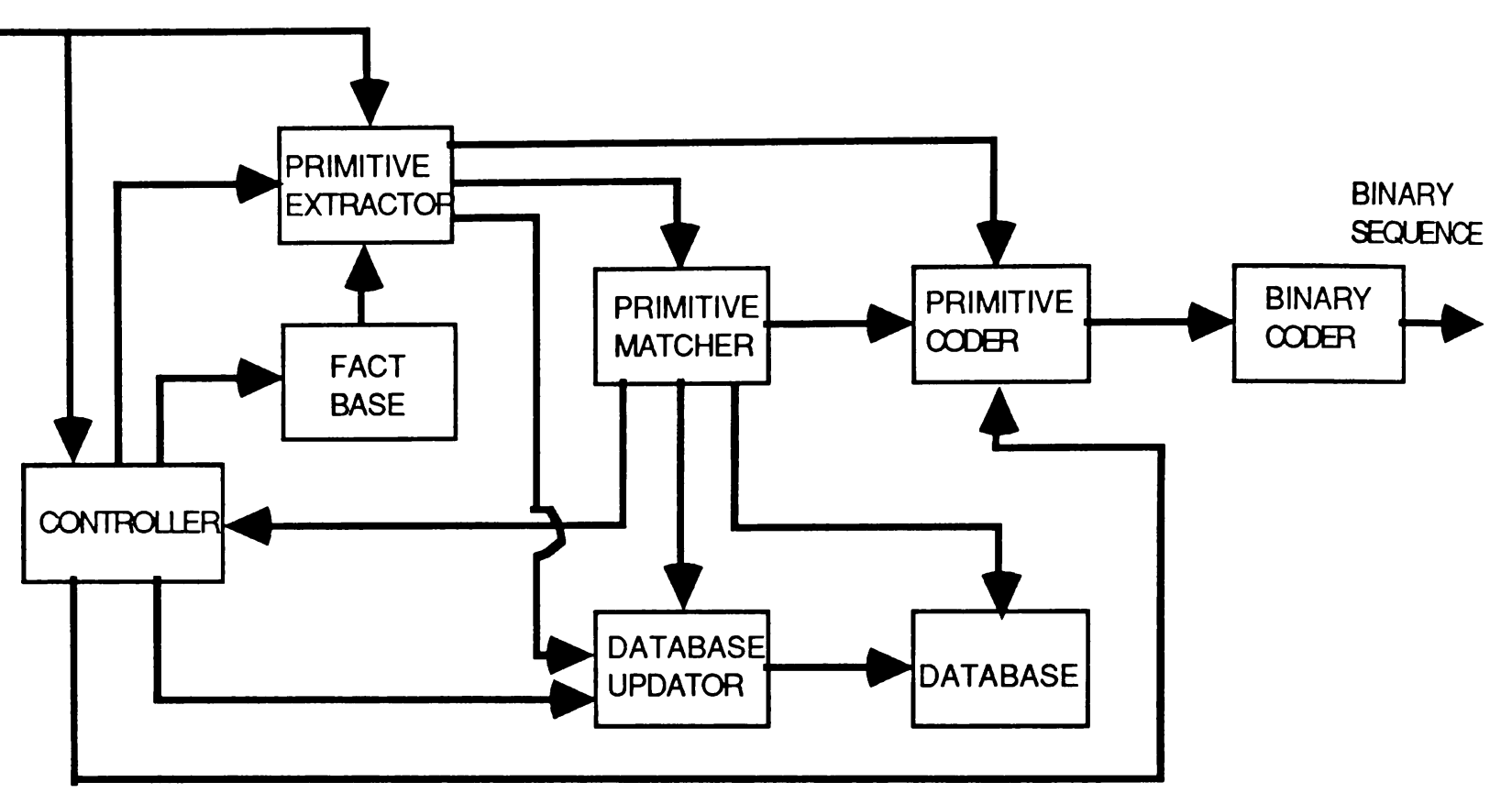

FIGURE 1: PRIMITIVE-BASED IMAGE CODER

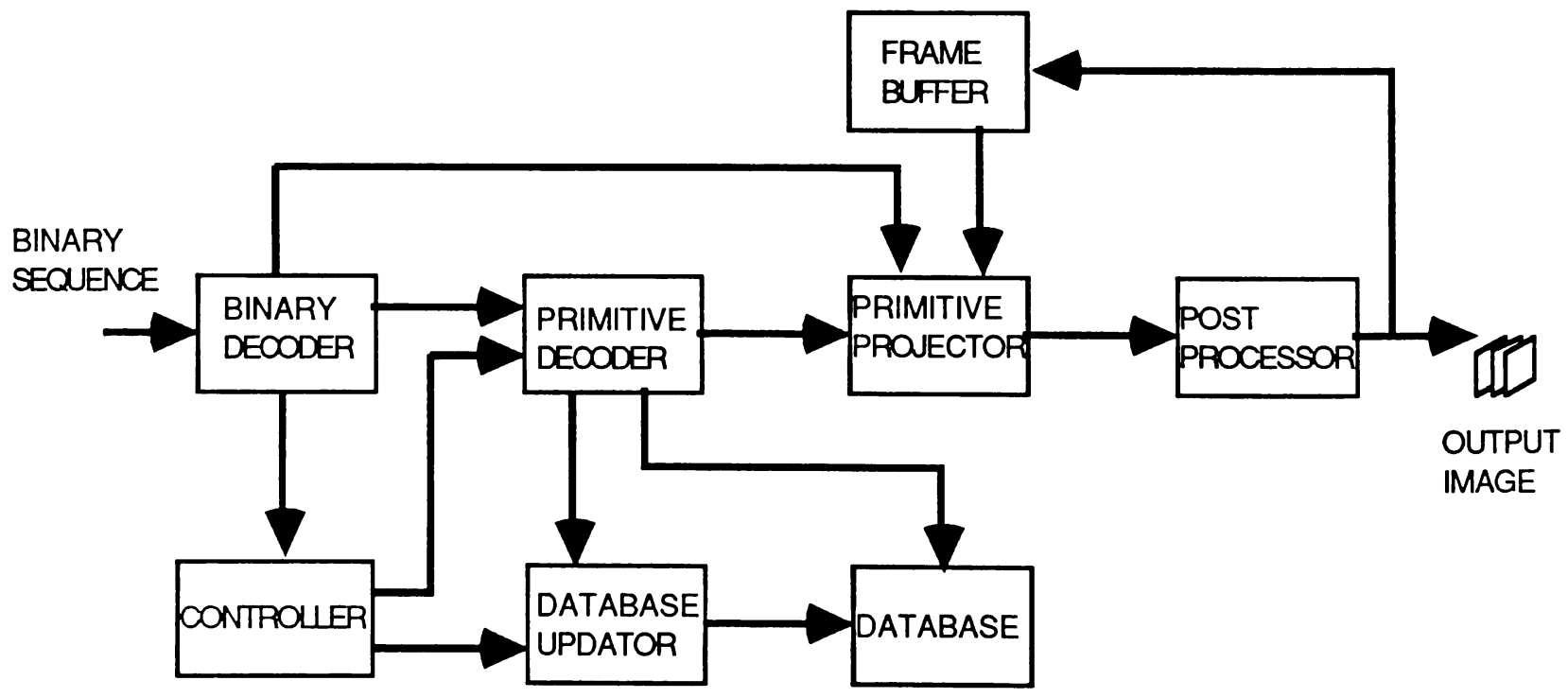

FIGURE 2: PRIMITIVE-BASED IMAGE DECODER 



Fig. 3. Relation of Angles $\alpha, \beta$, and $\theta$ to Head Position. 


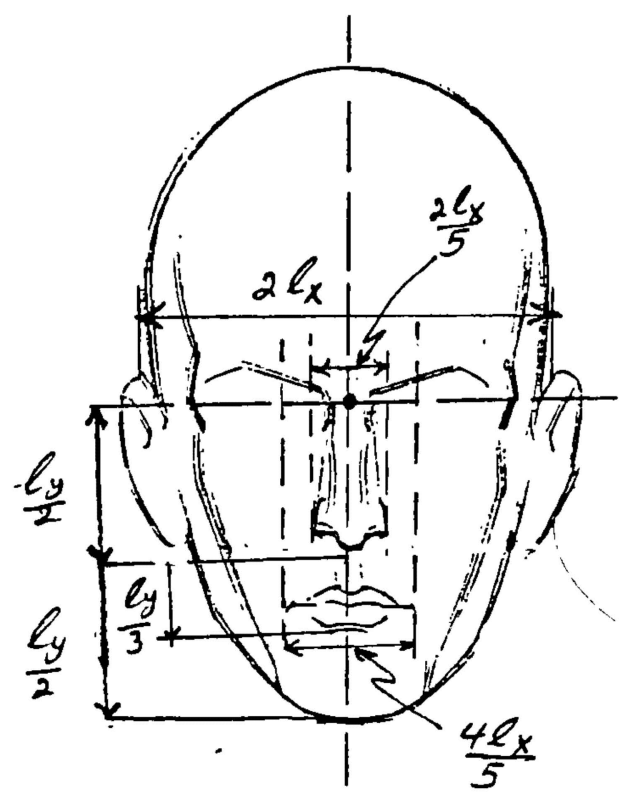

Fig. 4. Head Features Location From Front View.

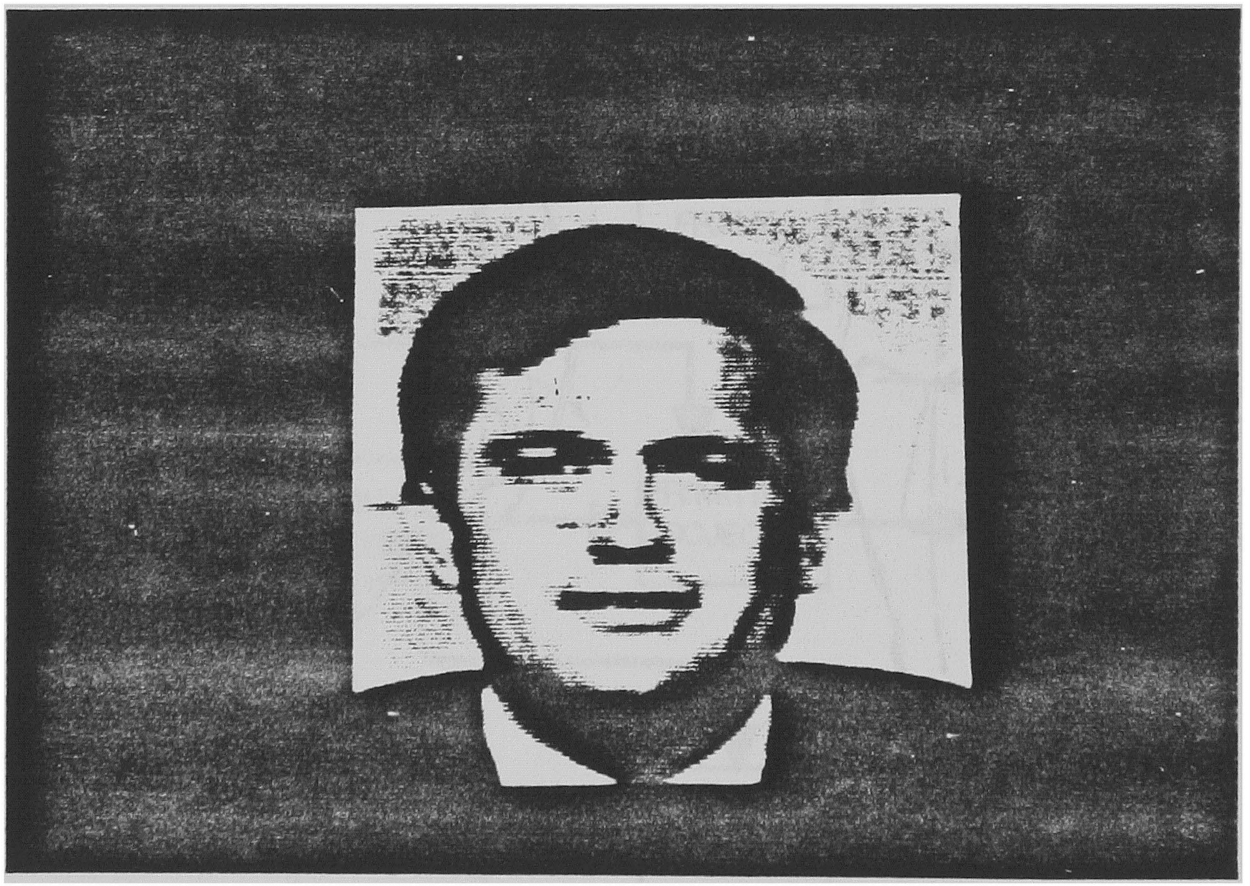

Fig. 5 128×128 Previous Frame 


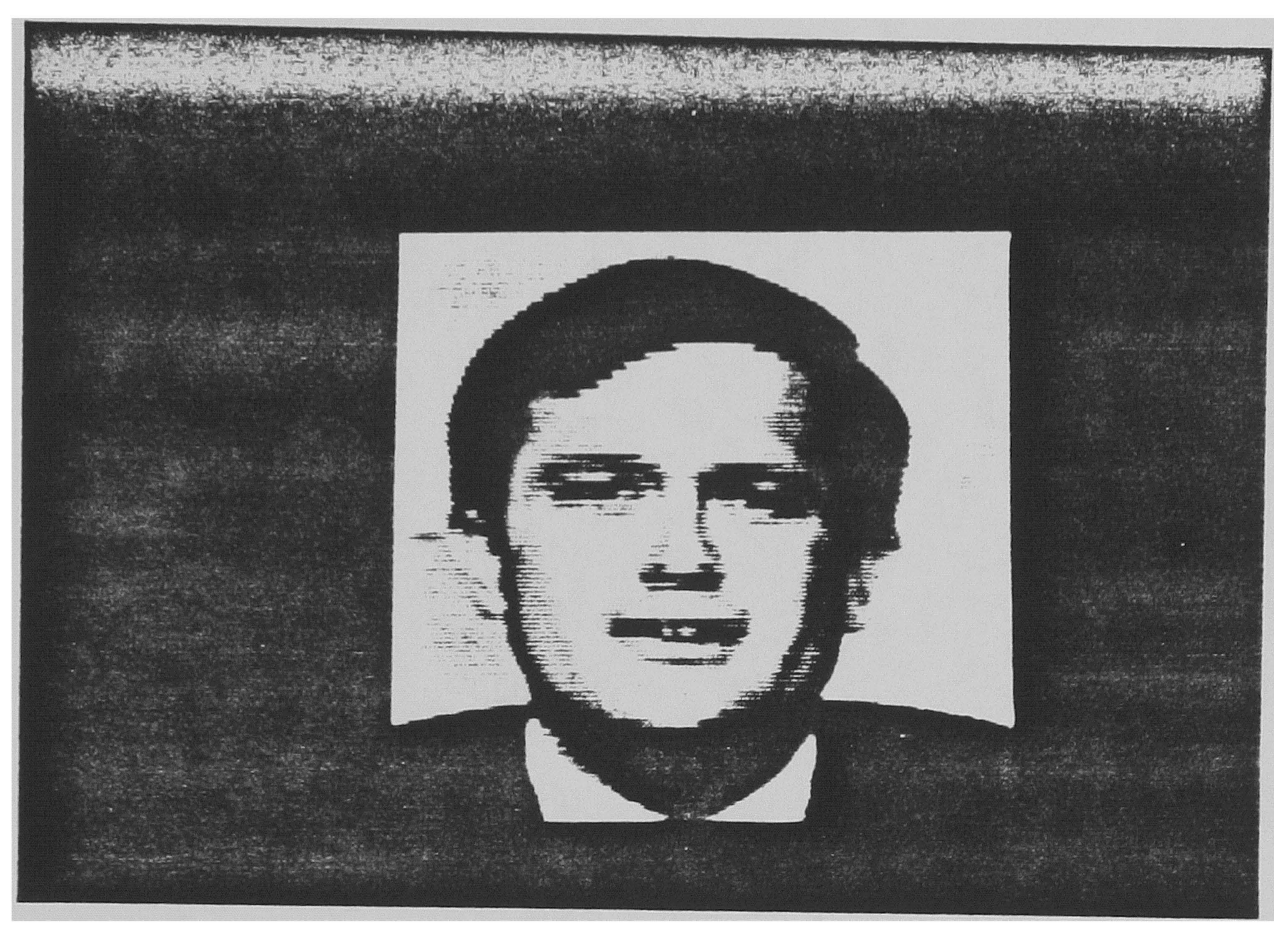

Fig. 6 128×128 Input Image To Be Coded

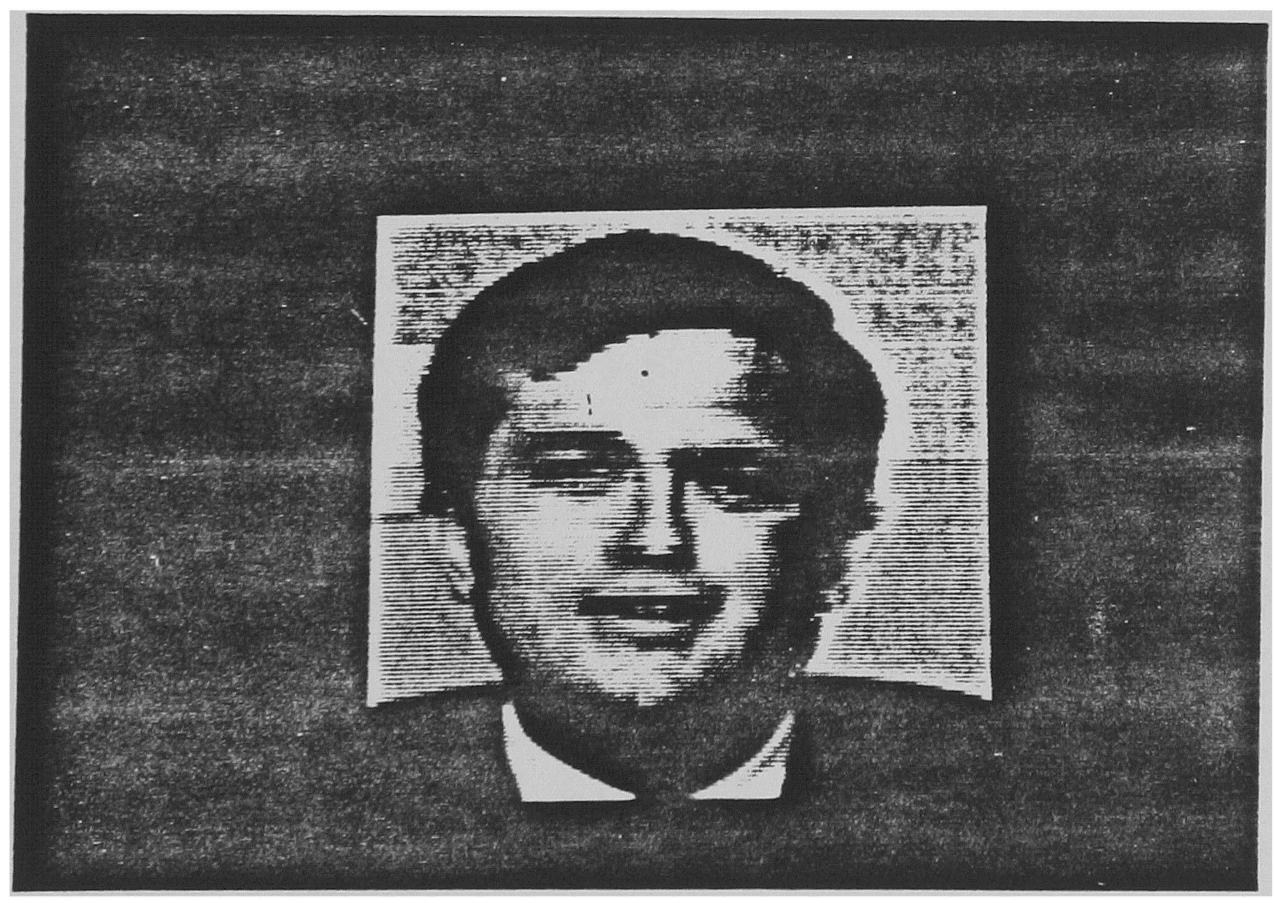

Fig. $7128 \times 128$ Decoded Output Image 